\title{
Tratamento das obstruções congênitas do ducto nasolacrimal
}

\author{
Management of congenital nasolacrimal duct obstruction
}

\author{
Epaminondas Castelo Branco Neto ${ }^{1}$ \\ Bruno Castelo Branco ${ }^{2}$ \\ Camila Correa Cardoso ${ }^{3}$ \\ Roberson Garcia de Carvalho ${ }^{4}$ \\ Eduardo Mota ${ }^{5}$ \\ André Castelo Branco ${ }^{6}$
}

Trabalho realizado no Serviço de Oftalmologia da Faculdade de Medicina da Universidade Federal da Bahia - UFBA - Salvador (BA) - Brasil.

${ }^{1}$ Professor Associado de Oftalmologia da Faculdade de Medicina da Universidade Federal da Bahia - UFBA Salvador (BA) - Brasil. Doutor pela Universidade Federal de São Paulo - UNIFESP - São Paulo (SP) - Brasil.

2 Professor Substituto de Oftalmologia da Faculdade de Medicina da UFBA - Salvador (BA) - Brasil. Doutor pela UNIFESP - São Paulo (SP) - Brasil.

${ }^{3}$ Oftalmologista pelo Servico de Oftalmologia da Faculdade de Medicina da UFBA - Salvador (BA) - Brasil.

${ }^{4}$ Oftalmologista pelo Serviço de Oftalmologia da Faculdade de Medicina da UFBA - Salvador (BA) - Brasil.

${ }^{5}$ Doutor e Professor Adjunto do Instituto de Saúde Coletiva da UFBA - Salvador (BA) - Brasil.

${ }^{6}$ Professor Adjunto de Oftalmologia da Faculdade de Medicina da UFBA - Salvador (BA) - Brasil. Doutor pela UNIFESP - São Paulo (SP) - Brasil.

Endereço para Correspondência: Epaminondas Castelo Branco Neto. Rua Horácio Urpia, 1 - Apto. 1.101 Salvador (BA) CEP 40150-250

Email: ecbranco@uol.com.br

Recebido para publicação em 16.03.2008

Última versão recebida em 18.12.2008

Aprovação em 20.12.2008

\begin{tabular}{|l|}
\hline RESUMO \\
\hline Objetivo: Apresentar os resultados do tratamento da obstrução naso- \\
lacrimal congênita com a massagem hidrostática de Crigler. Métodos: \\
Cento e oitenta e seis crianças com idade de até 32 meses com obstrução \\
nasolacrimal congênita foram estudadas retrospectivamente de 1990 a \\
2005. O procedimento foi repetido três vezes em cada sessão, com \\
intervalo de uma semana entre as sessões. A análise foi realizada com o \\
programa informatizado EPI INFO versão 6.04. A significância estatísti- \\
ca foi avaliada pela aplicação dos testes do qui-quadrado e Kruskal- \\
Wallis. Resultados: A faixa de idade predominante nos dois sexos foi de \\
1 a 5 meses (56,4\%), a localização foi bilateral em 36,3\%, do lado direito \\
em 34,1\% e do lado esquerdo em 29,6\%. A epífora estava presente em \\
$100 \%$ dos casos e secreção em 56,5\%. Curaram com uma sessão 43,6\% \\
dos casos, com duas 23,3\%, com três 17,4\% e com 4 e 5 ou mais sessões \\
$5,8 \%$ e 9,0\% dos pacientes, respectivamente. Os 14 pacientes que não \\
responderam a massagem foram tratados com sondagem. Conclusão: A \\
massagem hidrostática de Crigler é efetiva para a obstrução nasolacrimal \\
congênita.
\end{tabular}

Descritores: Obstrução dos ductos lacrimais; Ducto nasolacrimal; Massagem

\section{INTRODUÇÃO}

A obstrução nasolacrimal congênita (ONLC) é a anormalidade mais comum do aparelho lacrimal da criança ${ }^{(1-2)}$. Sua incidência varia de $1,8 \%$ a $20 \%{ }^{(1-3)}$. A causa mais freqüente é a imperfuração da membrana localizada na porção distal do conduto nasolacrimal ${ }^{(4-5)}$.

Testes realizados na primeira semana de vida sugerem que 54,3\% dos dutos nasolacrimais permanecem fechados ao nascer ${ }^{(6)}$. Entretanto, nem todos os pacientes que apresentam obstrução das vias lacrimais relatam quadro clínico ${ }^{(7)}$. A maioria se cura espontaneamente durante os primeiros meses de $\operatorname{vida}^{(6)}$.

Os sinais comuns de ONLC são epífora, secreção mucopurulenta e crosta nos cílios, aparecendo nas primeiras semanas de vida. Em alguns casos o diagnóstico é confirmado pela secreção que reflui dos orifícios lacrimais, após a compressão no saco lacrimal ${ }^{(8)}$.

O tratamento pode ser realizado com a massagem hidrostática de Crigler em crianças de até um ano de idade ${ }^{(9-16)}$. Uma segunda alternativa de tratamento é a sondagem lacrimal que pode ser realizada no consultório nos primeiros seis meses de vida, sem anestesia geral ou em crianças acima de seis meses no centro cirúrgico com anestesia geral ${ }^{(15,17-18)}$.

O objetivo deste trabalho é apresentar os nossos resultados com a massagem hidrostática de Crigler no tratamento de crianças com ONLC. 


\section{MÉTODOS}

Realizou-se um estudo retrospectivo, utilizando dados de prontuário de 186 crianças de atendidas, com idade de 6 dias até 32 meses de vida, entre 1990 e 2005, portadoras de obstrução congênita do conduto nasolacrimal (ONLC), clinicamente diagnosticada pela presença de epífora que se iniciou nas primeiras semanas de vida, associada ou não a presença de secreção mucopurulenta e crosta nos cílios. Todas as crianças foram examinadas e tratadas por um dos autores em sua clínica particular. Crianças com outras anomalias congênitas da face foram excluídas do estudo.

A massagem foi realizada por um dos autores (ECBN), como descrita por Crigler, colocando-se o indicador sobre o canalículo comum para bloquear a saída de material através do mesmo e comprimindo o saco lacrimal firmemente para baixo para aumentar a pressão hidrostática e provocar a desobstrução do conduto nasolacrimal ${ }^{(9)}$. $\mathrm{O}$ movimento era realizado três vezes em cada sessão.

As massagens foram repetidas semanalmente e não se fez uso de antibiótico tópico durante o tratamento. Todas as crianças foram tratadas até o desaparecimento dos sintomas ou até que completassem um ano de idade. Foi considerado cura espontânea a criança com história compatível com o diagnóstico de obstrução das vias lacrimais, mas com as vias lacrimais pérvias no primeiro exame. As crianças que não se curaram com as massagens, foram submetidas à sondagem sob anestesia geral.

Os dados para a análise incluíram: idade, sexo, lateralidade (lado direito, lado esquerdo e ambos os lados), presença de lacrimejamento, presença de secreção, utilização de colírio, realização de sondagem, número de sessões de massagem e cura espontânea. Os dados foram preparados e a análise realizada com o programa informatizado EPI INFO versão 6.04, efetuando-se a consolidação e a representação tabular e gráfica dos dados. A significância estatística foi testada pela aplicação do teste do qui-quadrado (com correção de Yates para pequenas frequências) para a comparação de proporções e do teste Kruskal-Wallis para a comparação de médias, adotandose nível de significância de 5\%.

\section{RESULTADOS}

A idade das 186 crianças variou de 6 dias de vida a 32 meses. A distribuição segundo sexo não mostrou diferença, sendo $54,5 \%$ das crianças (102) do sexo feminino. Para os menores de 1 mês a média de idade foi de 19,2 dias (desvio padrão ( $\mathrm{DP}=5,8)$ e mediana $=19,5$. Para os maiores de 1 mês, a média de idade foi igual a 4,98 meses ( $D P=5,2)$ e mediana de 3,0 meses. A faixa de idade predominante foi a de 1 a 5 meses $(56,4 \%)$ em ambos os sexos (Tabela 1$)$.

Observaram-se as seguintes frequências da apresentação de ONLC por localização: ambos os lados em 36,3\%, lado direito em $34,1 \%$ e lado esquerdo em $29,6 \%$ (Tabela 2). A distribuição da localização por faixa etária apresentou-se dife-

\begin{tabular}{|c|c|c|c|c|c|c|}
\hline \multirow{3}{*}{ Faixa etária } & \multicolumn{4}{|c|}{ Sexo } & \multirow{2}{*}{\multicolumn{2}{|c|}{ Total }} \\
\hline & \multicolumn{2}{|c|}{ Masc. } & \multicolumn{2}{|c|}{ Fem. } & & \\
\hline & № & $\%$ & № & $\%$ & № & $\%$ \\
\hline 6 a 15 dias & 5 & 45,5 & 6 & 54,5 & 11 & 6,1 \\
\hline 16 a 28 dias & 11 & 47,8 & 12 & 52,2 & 23 & 12,7 \\
\hline 1 a 5 meses & 48 & 47,1 & 54 & 52,9 & 102 & 56,4 \\
\hline 6 a 11 meses & 11 & 35,5 & 20 & 64,5 & 31 & 17,1 \\
\hline 12 a 32 meses & 7 & 50,0 & 7 & 50,0 & 14 & 7,7 \\
\hline Total & 82 & 45,3 & 99 & 54,7 & 181 & 100,0 \\
\hline
\end{tabular}

\begin{tabular}{|c|c|c|}
\hline \multirow[t]{2}{*}{ Características } & \multicolumn{2}{|c|}{ Frequência } \\
\hline & № & $\%$ \\
\hline \multicolumn{3}{|l|}{ Localização $(n=179)$} \\
\hline Ambos os lados & 65 & 36,3 \\
\hline Lado direito & 61 & 34,1 \\
\hline Lado esquerdo & 53 & 29,6 \\
\hline \multicolumn{3}{|l|}{ Sinais } \\
\hline \multicolumn{3}{|l|}{ Com secreção } \\
\hline Total $(n=164)$ & 105 & 56,5 \\
\hline Em AO & 39 & 60,0 \\
\hline Em OD & 30 & 49,2 \\
\hline Em OE & 32 & 60,4 \\
\hline \multicolumn{3}{|l|}{ Tratamento } \\
\hline Colírio sim $(n=176)$ & 128 & 72,7 \\
\hline Antibiótico tópico sim & 127 & 68,3 \\
\hline $\begin{array}{l}\text { Sondagem sim } \\
\text { para idade * }\end{array}$ & 14 & 7,5 \\
\hline 1 a 11 meses $(n=134)$ & 8 & 6,0 \\
\hline 12 a 32 meses $(n=14)$ & 6 & 42,9 \\
\hline
\end{tabular}

rente nos diversos grupos, embora as diferenças entre proporções não tenham sido estatisticamente significativas. Para os menores de 1 mês ocorreu predominância de ambos os lados $(42,4 \%)$. A partir de 1 mês de vida, a proporção de casos de lado direito diminuiu com a idade, ao passo que a proporção de pacientes de lado esquerdo aumentou, alcançando o máximo de $46,2 \%$ no grupo com idade de 12 a 32 meses (Gráfico 1).

Todas as crianças apresentaram lacrimejamento. Secreção mucopurulenta, entretanto, só foi vista em 56,5\% (105) dos casos, sendo menor a proporção de pacientes com este sinal $(49,2 \%)$ naqueles com a apresentação no lado direito (Tabela 2).

Massagem foi realizada em todos os pacientes. Para 43,6\% (75) dos casos ocorreu resolução na primeira sessão de massagem. Outros $40(23,3 \%)$ necessitaram de duas sessões de massagem, $30(17,4 \%)$ realizaram três sessões, e os casos com 4 e 5 ou mais sessões representaram, $5,8 \%$ e $9,9 \%$ dos pacientes, respectivamente.

A média de sessões de massagem por faixa etária variou pouco, com intervalo de 1,7 a 2,6 e diferença estatisticamente 
não significativa entre os valores para os pacientes com idade igual ou maior que 1 mês (Tabela 3 ).

Naqueles que não responderam satisfatoriamente às sessões de massagem foi realizada sondagem o que representou $7,5 \%$ do total (Tabela 2). Os 14 pacientes que necessitaram sondagem tiveram idade igual ou maior que 1 mês, com distribuição desses casos por idade apresentando frequência de $6 \%$ e $42,9 \%$ (6) nas faixas de 1 a 11 meses e de 12 a 32 meses, com diferença estatisticamente significativa (Tabela 2).

Por apresentação a distribuição desses casos foi: $35,7 \%$ para ambos os lados e lado direito em igual proporção e 28,6\% para lado esquerdo. Seis casos $(42,9 \%)$ submetidos à sondagem realizaram previamente uma sessão de massagem e os outros em igual proporção realizaram 2 a 3 sessões. A diferença entre as médias do número de sessões de massagem do grupo sem sondagem $(2,4 \pm 2,1)$ e do grupo submetido a este procedimento $(2,9 \pm 3,1)$ não foi estatisticamente significativa (teste Kruskal-Wallis, $\mathrm{p}=0,6358$ ).

Em 11 pacientes $(5,9 \%)$ foi observada cura espontânea. Nesses casos, $3(27,3 \%)$ ocorreram entre os menores de 1 mês de vida e $6(54,5 \%)$ no grupo de 1 a 5 meses de idade. A distribuição desses pacientes não diferiu significativamente da proporção do total de casos por faixa etária. Pouco mais da metade (6) ocorreu na apresentação bilateral.

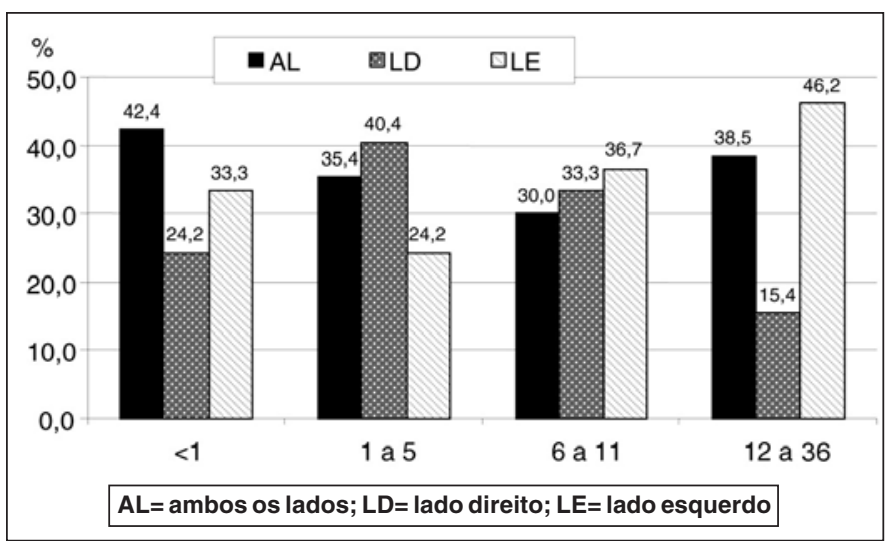

Gráfico 1 - Distribuição porcentual dos pacientes com obstrução nasolacrimal congênita por localização (AL, LD, LE) e faixa etária (meses), Salvador, $1990-2005$

\begin{tabular}{|c|c|c|c|c|}
\hline Faixa etária & № & Média & Desvio padrão & Mediana \\
\hline 6 a 15 dias & 11 & 2,4 & 2,1 & 2,0 \\
\hline 16 a 28 dias & 23 & 2,4 & 1,8 & 2,0 \\
\hline 1 a 5 meses & 103 & 2,6 & 2,4 & 2,0 \\
\hline 6 a 11 meses & 31 & 1,9 & 1,0 & 2,0 \\
\hline 12 a 32 meses & 14 & 1,7 & 1,0 & 1,0 \\
\hline
\end{tabular}

\section{DISCUSS ÃO}

Este estudo confirma após avaliar 186 pacientes durante 15 anos, que a massagem hidrostática de Crigler é útil no tratamento das obstruções congênitas do ducto nasolacrimal. Houve resolução do quadro em $92,5 \%$ dos casos. Os bons resultados apresentados foram obtidos sem o uso de antibióticos tópicos durante as massagens. Os pacientes que fizeram uso dos antibióticos o fizeram antes do início das massagens.

Em 1923, Crigler propôs a técnica da massagem do saco lacrimal, com a qual obteve $100 \%$ de sucesso durante $7 \operatorname{anos}^{(9)}$. Utilizando a mesma técnica de Crigler, outros obtiveram cura de $87,5 \%$ e $94,7 \%{ }^{(11-12)}$. Há relato de que quando a epífora se inicia antes dos 2 meses de vida, há maior chance de cura com a massagem $^{(10)}$.

Estudo prospectivo randomizado comparou 3 grupos de portadores de ONLC: grupo controle com 58 crianças, um segundo grupo com 59 crianças tratadas com a massagem hidrostática de Crigler e um terceiro grupo com 58 crianças tratadas com massagem realizadas por familiares e concluiu que a massagem hidrostática de Crigler é o método mais efetivo ${ }^{(11)}$.

Segundo outros autores, com a massagem hidrostática de Crigler a cura aconteceu em 93,3\% de 30 crianças de 1 a 12 meses e $79,3 \%$ de 29 crianças de 13 a 24 meses $^{(14)}$, mostrando que a idade das crianças deve influenciar no resultado do tratamento. $\mathrm{O}$ estudo atual, assim como a literatura também demonstra que quando a ONLC é diagnosticada até o primeiro ano de vida apresenta um alto índice de resolução.

Apesar dos altos índices de sucesso com a massagem de Crigler, outros autores relataram sucesso total com o método em apenas $45 \%$ das crianças de até 1 ano, $56 \%$ dos com menos de 2 meses de idade, diminuindo para $46 \%$ de 2 a 6 meses e para $28 \%$ com mais de 6 meses de idade ${ }^{(15)}$. Neste estudo a resolução com uma massagem foi obtida em $43,6 \%$ dos casos e em $66,9 \%$ com até duas massagens e em $87,3 \%$ dos casos com até três massagens ${ }^{(15)}$. A idade influiu significativamente na chance do paciente se submeter à sondagem, sendo realizada em apenas $6 \%$ dos pacientes com tratamento iniciado com menos de 1 ano de idade e em 42,9\% dos pacientes entre $12 \mathrm{e}$ 32 meses $^{(15)}$.

Neste estudo as crianças com faixa etária de 6 a 15 dias, 16 a 28 dias, 1 a 5 meses, 6 a 11 meses e de 12 a 32 meses o número médio de massagens necessárias foi de: 2,4; 2,4; 2,6;1,9 e 1,7, respectivamente. Esta diferença ocorreu porque após um ano de idade o autor não realizava mais de duas massagens, passando para outra fase do tratamento.

Todos os nossos pacientes apresentaram lacrimejamento e 164 apresentaram também secreção. Dos 176, os 127 (68\%) que fizeram uso de colírio o fizeram por orientação prévia, uma vez que durante nosso tratamento não recomendamos o uso do colírio. Dos 186 casos, 14 não responderam ao tratamento com massagem e curaram com apenas uma sondagem. Outros autores descreveram o resultado da sondagem de 2.369 condutos nasolacrimais obstruídos. Destes, 1.986 tinham menos 
de 9 meses de idade tendo obtido cura com uma sondagem em $94 \%$ (1.866) e 383 com mais de 9 meses obtiveram $83 \%$ de cura com uma sondagem. Dos 186 que permaneceram obstruídos após a primeira sondagem 102 (55\%) curaram com uma segunda sondagem ${ }^{(17)}$.

Outro estudo, de 1986, demonstrou que ao sondarem 138 vias lacrimais em crianças com mais de 13 meses de idade a taxa de sucesso foi de $93,5 \%$ com uma sondagem e $100 \%$ após duas sondagens ${ }^{(18)}$. Entretanto outros estudos ao sondarem uma ou mais vezes 334 vias lacrimais obstruídas em crianças de 1 mês a 10 anos de idade obtiveram sucesso em 75,53\% em crianças até 35 meses de idade e de $68,56 \%$ no grupo total ${ }^{(15)}$.

\section{CONCLUSÃO}

Este estudo confirma após avaliar 186 pacientes durante 15 anos, que a massagem hidrostática de Crigler é útil no tratamento das obstruções congênitas do ducto nasolacrimal. Houve resolução do quadro em 92,5\% dos casos. Os bons resultados apresentados foram obtidos sem o uso de antibióticos tópicos durante as massagens. Os pacientes que fizeram uso dos antibióticos o fizeram antes do início das massagens.

\section{ABSTRACT}

Purpose: To present the success rate in treating congenital nasolacrimal duct obstruction with hydrostatic pressure (Crigler method). Methods: Clinical data from 186 children aged up to 32 months treated from 1990 until 2005 were reviewed retrospectively. The procedure was repeated up to 3 times at a one week interval. Using EPI INFO version 6.04 software for analysis, data were tabulated and statistical significance was evaluated by applying chi-square and KruskalWallis tests. Results: Leading age group was 1 to 5 months old $(56.4 \%)$ for males and females. Affection was bilateral in $36.3 \%, 34.1 \%$ on right side and $29.6 \%$ on the left side. All cases had epiphora and $56.5 \%$ presented mucopurulent discharge. Cure was achieved with one massage for $43.6 \%, 23.3 \%$ with two, $17.4 \%$ with three and, with 4 and $5+$ sessions in respectively $5.8 \%$ and $9.0 \%$. Those 14 cases who did not respond to massage were treated with probing. Conclusion: The Crigler method was effective in managing congenital nasolacrimal duct obstruction cases.

Keywords: Lacrimal duct obstruction; Nasolacrimal duct; Massage

\section{REFERÊNCIAS}

1. Guerry D, Kending EL Jr. Congenital impatency of the nasolacrimal duct. Arch Ophthalmol. 1948;39(2):193-204.

2. MacEwen CJ, Young JD. Epiphora during the first year of life. Eye. 1991; 5(PT 5):596-600

3. Dias AK, Soccol O, Cunha M, Gomes JA, Pinheiro RK, Peres MF. Frequência de obstrução congênita do ducto nasolacrimal na clínica pediátrica da Santa Casa de São Paulo. Arq Bras Oftalmol. 1994;57(2):118-21.

4. Cassidy JV. Dacryocystitis in infancy. Am J Ophthalmol. 1948;31(7):773-80; disc, 875-7.

5. Silva JA. Tratamento das dacriocistites congênitas na Infância. Pediatr Atual. 1992;5(1):45-6

6. Korchmaros I, Szalay E, Fodor M, Jablonszky E. Spontaneous opening rate of congenitally blocked Nasolacrimal ducts. In: Yamaguchi, M., editor. Recent advances on the lacrimal system. Tokyo: Asahi Evening News; 1978. p.30-5.

7. Schellini SA, Hoyama E, Rocha MC, Rugollo A Jr, Padovani CR. Evaluation of lacrimal system outflow in the newborn by dye tests. Orbit. 2000;19(3): 137-42.

8. Cassidy JV. Developmental anatomy of the nasolacrimal duct. AMA Arch Ophthalmol. 1952;47(2):141-58.

9. Crigler LW. The treatment of congenital dacryocystitis. JAMA. 1923;81:23-4.

10. Schellini SA, Viveiros MM, Jaqueta E, Padovani CR, Padovani CR. Obstrução nasolacrimal congênita: fatores relacionados com a possibilidade de cura. Arq Bras Oftalmol. 2005;68(2):241-4.

11. Nelson LB, Calhoun JH, Menduke H. Medical management of congenital nasolacrimal duct obstruction. Ophthalmology. 1985;92(9):1187-90.

12. Castelo Branco Neto E, Castelo Branco AB, Castelo Branco B, Marback EF. Tratamento da obstrução congênita do conduto nasolacrimal. Arq Bras Oftalmol. 1994;57(4):248-51.

13. Kushner BJ. Congenital nasolacrimal system obstruction. Arch Ophthalmol. 1982;100(4):597-600

14. Nucci P, Capoferri C, Alfarano R, Brancato R. Conservative management of congenital nasolacrimal duct obstruction. J Pediatr Ophthalmol Strabismus. 1989;26(1):39-43.

15. Chaim MP, Bison SH, Silva JA. Obstrução congênita do duto lacrimonasal: estudo de 284 pacientes. Arq Bras Oftalmol. 1996;59(5):519-21.

16. Stolovitch C, Michaeli A. Hydrostatic pressure as an office procedure for congenital nasolacrimal duct obstruction. J AAPOS. 2006;10(3):269-72.

17. Stager D, Baker JD, Frey T, Weakley DR Jr, Birch EE. Office probing of congenital nasolacrimal duct obstruction. Ophthalmic Surg. 1992;23(7):482-4.

18. El-Mansoury J, Calhoun JH, Nelson LB, Harley RD. Results of late probing for congenital nasolacrimal duct obstruction. Ophthalmology. 1986;93(8): $1052-4$ 